\title{
Role of neutron rearrangement channels in sub-barrier fusion
}

\author{
A. V. Karpov ${ }^{1, a}$, V. A. Rachkov ${ }^{1}$, A. S. Denikin ${ }^{2,1}$, and V. I. Zagrebaev ${ }^{1}$ \\ ${ }^{1}$ Flerov Laboratory of Nuclear Reactions, JINR, Dubna, 141980 Russia \\ ${ }^{2}$ International University "Dubna", Dubna, 141980 Russia
}

\begin{abstract}
Different factors influencing the sub-barrier fusion enhancement owing to neutron rearrangement with positive $Q$ values are studied. It was found that opposite to the previous opinion the presence of positive $Q$ values is necessary but not sufficient to observe enhancement of the sub-barrier fusion. "Rigidity" of colliding nuclei with respect to collective excitations plays a crucial role for the sub-barrier fusion enhancement due to neutron rearrangement. Neutron binding energy has a strong impact but only in the case of fusion of light nuclei.
\end{abstract}

\section{Motivation}

Sub-barrier fusion is one of the intensively studied phenomena, that is still far from its complete understanding. The sub-barrier fusion enhancement induced by coupling of relative motion to surface deformations or rotation of heavy deformed nuclei is well understood and properly described within the quantum coupled channel approach (see e.g. [1-3]) and within the empirical coupled channel (ECC) model [4]. At the same time there are many experimental evidences of additional enhancement of the sub-barrier fusion cross section due to neutron rearrangement with positive $Q$ values. The mechanism of sequential fusion was proposed in [5] which described for the first time an additional sub-barrier fusion enhancement owing to neutron rearrangement with positive $Q$ value at approaching stage. The corresponding model (called the ECC model with neutron rearrangement) is used in this work. The mechanism of influence of neutron rearrangement on the sub-barrier fusion is connected with the fact that the spreading of the valence neutron's wave function into the volume of the other nucleus takes place before the colliding nuclei overcome the Coulomb barrier $[6,7]$, and, therefore, neutron rearrangement at approaching stage may really influence the sub-barrier fusion dynamics giving a gain in kinetic energy of colliding nuclei if it occurs with positive $Q$ value. This effect can be easier observed if one compares the sub-barrier fusion cross sections for two close projectile-target combinations for one of which neutron rearrangement with positive $Q$ values is possible whereas for another one all neutron transfers have negative $Q$ values. The pair of reactions ${ }^{40} \mathrm{Ca}+{ }^{96} \mathrm{Zr}$ and ${ }^{40} \mathrm{Ca}+{ }^{90} \mathrm{Zr}$ is "classical" example of such kind [see Fig. 1 (a)]. Coupling of relative motion to the surface vibrations explains the observed cross section for the ${ }^{40} \mathrm{Ca}+{ }^{90} \mathrm{Zr}$ reaction, but it is insufficient to describe additional sub-

\footnotetext{
ae-mail: karpov@jinr.ru
}


Figure 1. (a) Fusion cross sections for ${ }^{40} \mathrm{Ca}+{ }^{96} \mathrm{Zr}$ (open circles [8] and filled squares [9]) and ${ }^{40} \mathrm{Ca}+{ }^{90} \mathrm{Zr}$ (filled circles [8]). The no-coupling limits are shown by the dotted curves. The dashed curves show the calculations with coupling to surface vibrations and without neutron transfer, whereas the solid line was obtained with additional accounting for neutron rearrangement. (b) The same but for ${ }^{4,6} \mathrm{He}+{ }^{64} \mathrm{Zn}$. Experimental data are from [10].

barrier fusion enhancement for the ${ }^{40} \mathrm{Ca}+{ }^{96} \mathrm{Zr}$ caused by neutron rearrangement. Another example of strong enhancement owing to neutron rearrangement is fusion of neutron rich weakly bound nuclei. Figure 1 (b) shows the fusion cross sections for two systems: ${ }^{4} \mathrm{He}+{ }^{64} \mathrm{Zn}$ (all $Q_{x n}$ 


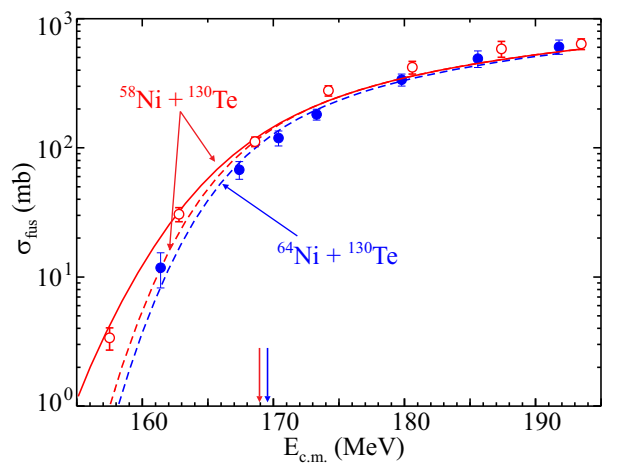

Figure 2. Fusion cross sections for ${ }^{58} \mathrm{Ni}+{ }^{130} \mathrm{Te}$ and ${ }^{64} \mathrm{Ni}+{ }^{130} \mathrm{Te}$. The experimental data [11] are shown by open and filled circles, respectively. The solid and dashed curves show the ECC calculations with and without neutron rearrangement, respectively.

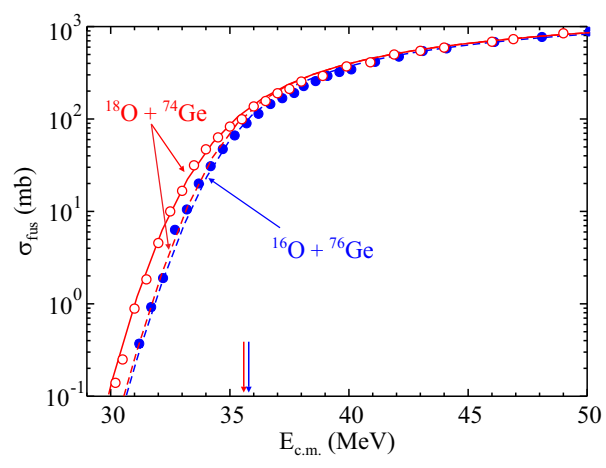

Figure 3. The same as in Fig. 2 but for the ${ }^{18} \mathrm{O}+{ }^{74} \mathrm{Ge}$ and ${ }^{16} \mathrm{O}$ $+{ }^{76} \mathrm{Ge}$ systems. The experimental data are from [12].

values are negative) and ${ }^{6} \mathrm{He}+{ }^{64} \mathrm{Zn}$ having large $Q_{x n}$ values $\left(Q_{1 n}=6.11 \mathrm{MeV}, Q_{2 n}=18.06 \mathrm{MeV}\right.$, and $Q_{3 n}=4.54$ $\mathrm{MeV})$. The enhancement owing to neutron rearrangement reaches of about factor 20 already at $2 \mathrm{MeV}$ below the Coulomb barrier and it is predicted even stronger at deeper sub-barrier energies.

It turns out that the role of neutron transfer in subbarrier fusion reactions is not completely clear. Recently several projectile-target combinations were reported (for example, ${ }^{130} \mathrm{Te}+{ }^{58,64} \mathrm{Ni}[11],{ }^{16,18} \mathrm{O}+{ }^{76,74} \mathrm{Ge}$ [12], ${ }^{60,64} \mathrm{Ni}+{ }^{100} \mathrm{Mo}$ [13]) for which the measured fusion cross sections do not demonstrate noticeable enhancement at sub-barrier energies in spite of positive $Q$ values for neutron rearrangement shown in Tab. 1. The corresponding fusion cross sections and the results of our analysis are shown in Figs. 2, 3 and 4. In all the cases neutron rearrangement with positive $Q$ values is taken into account. However only in the case of the ${ }^{58} \mathrm{Ni}+{ }^{130} \mathrm{Te}$ reaction some excess in the sub-barrier fusion cross section is visible as compared with more neutron rich system ${ }^{64} \mathrm{Ni}+{ }^{130} \mathrm{Te}$ having all negative $Q_{x n}$-values except $Q_{2 n}=+0.55 \mathrm{MeV}$. Note that the model used takes into account neutron rearrangement, reproduces quite well the experimental data and also does not predict any sub-barrier fusion enhancement for these specific reactions with positive $Q$ values of neutron transfers. Thus, we have to understand what features of

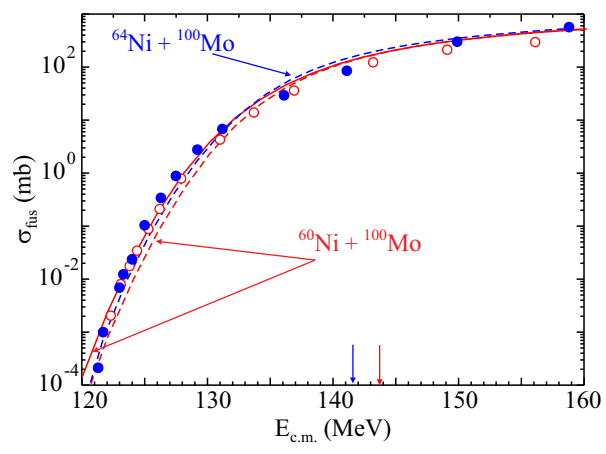

Figure 4. The same as in Fig. 2 but for the ${ }^{60} \mathrm{Ni}+{ }^{100} \mathrm{Mo}$ and ${ }^{64} \mathrm{Ni}+{ }^{100} \mathrm{Mo}$ systems. The experimental data are from [13].

these reactions (properties of colliding nuclei) suppress a gain coming from positive $Q$ value neutron rearrangement (clearly visible in many other cases).

The main purpose of this work is to find the other factors (conditions) of neutron rearrangement with positive $Q$ value which favour (or prevent) sub-barrier fusion enhancement. All the presented calculations have been performed (and can be easily repeated) with the NRV Fusion code allocated at the web site with free access [3].

Table 1. $Q_{x n}$ values of neutron transfer and vibrational properties of the targets. The energies are given in $\mathrm{MeV}$.

\begin{tabular}{l|cc|cc}
\hline \hline Reaction & $1 \mathrm{n}$ & $2 \mathrm{n}$ & $E_{2^{+}}$ & $\left\langle\beta_{2}^{0}\right\rangle$ \\
\hline${ }^{40} \mathrm{Ca}+{ }^{96} \mathrm{Zr}$ & +0.51 & +5.53 & 1.75 & 0.080 \\
${ }^{58} \mathrm{Ni}+{ }^{130} \mathrm{Te}$ & +0.58 & +5.89 & 0.84 & 0.118 \\
${ }^{60} \mathrm{Ni}+{ }^{100} \mathrm{Mo}$ & -0.47 & +4.20 & 0.54 & 0.231 \\
${ }^{18} \mathrm{O}+{ }^{74} \mathrm{Ge}$ & -1.54 & +3.74 & 0.60 & 0.283 \\
\hline \hline
\end{tabular}

\section{Interplay of nuclear properties and sub-barrier fusion enhancement}

In this section different factors influencing the enhancement of the sub-barrier fusion due to neutron rearrangement are discussed.

\section{1 $Q$ values}

The $Q$ values of neutron transfer as the factor determining the enhancement of the sub-barrier fusion cross section was discussed many times (see, e.g., $[5,14]$ ). We repeat here the main points. The statement is that if the system of two colliding nuclei has positive $Q$ values of neutron transfer then one may expect that the sub-barrier fusion cross section will demonstrate enhancement due to neutron rearrangement additional to the one caused by the coupling of the relative motion to vibrational and/or rotational degrees of freedom.

The last but not least point is that only the rearrangement of outermost neutrons (normally $1 \mathrm{n}$ and $2 \mathrm{n}$ transfer channels) may enhance the sub-barrier fusion significantly. This happens because the coupling to neutron rearrangement channels influences the fusion probability only 


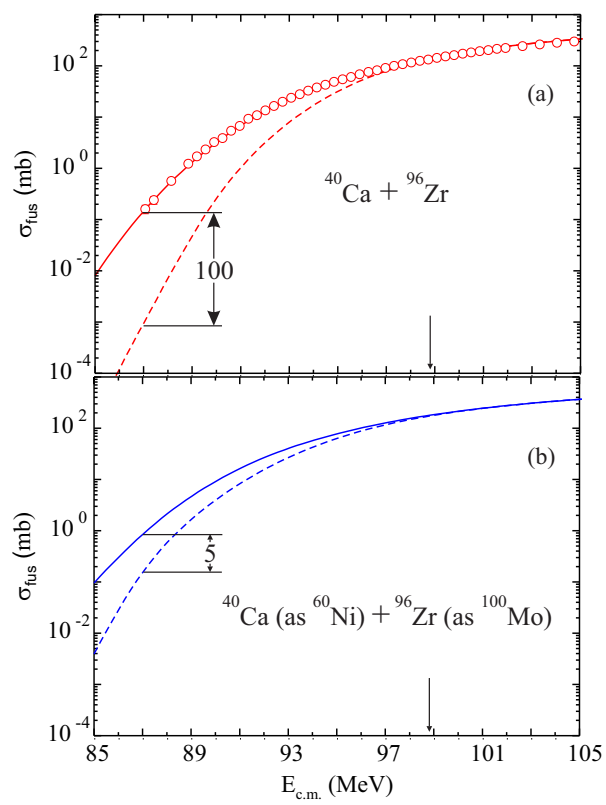

Figure 5. The fusion cross sections for the ${ }^{40} \mathrm{Ca}+{ }^{96} \mathrm{Zr}$ system (a) and for the same system but with the vibrational properties of ${ }^{40} \mathrm{Ca}$ and ${ }^{96} \mathrm{Zr}$ being substituted by those of ${ }^{60} \mathrm{Ni}$ and ${ }^{100} \mathrm{Mo}$, respectively (b). The solid and dashed curves show the results of the ECC calculations with and without accounting for neutron rearrangement channels, respectively.

if such rearrangement takes place before overcoming the Coulomb barrier. Therefore, only a few valence neutrons having largest radii of their wave functions should be taken into account in the analysis of the sub-barrier fusion.

\subsection{Properties of collective excitations}

The influence of the collective properties of nuclei on the enhancement of the fusion cross section due to neutron rearrangement is illustrated in Fig. 5. The statement is that the fusion enhancement due to neutron rearrangement is larger for the systems having smaller fusion enhancement due to the coupling to collective states. Figure 5 (a) shows the fusion cross sections for the ${ }^{40} \mathrm{Ca}+{ }^{96} \mathrm{Zr}$ system. The fusion enhancement due to neutron rearrangement constitutes two orders of magnitude at the energies $12 \mathrm{MeV}$ below the barrier (compare the solid and dashed curves). Both reaction partners are magic spherical nuclei, and, therefore, they are hardly deformed nuclei, since, their first excited states (see Table 1) are rather high. According to our conclusion this motivates so large influence of neutron rearrangement on the sub-barrier fusion. If now we assume that these nuclei are softer with respect to their deformation than they actually are, and replace the vibrational properties of ${ }^{40} \mathrm{Ca}$ and ${ }^{96} \mathrm{Zr}$ by those of ${ }^{60} \mathrm{Ni}$ and ${ }^{100} \mathrm{Mo}$ (softer nuclei) the influence of the collective excitations increases [see Fig. 5 (b)]. As the result, account for neutron transfer channels gives now additional enhancement factor 5 instead of 100 . Note, that in these calculations we alter only vibrational properties of nuclei and do not change the rest model parameters (potential, charges, masses, binding energies, $Q$ values, etc.).
The performed analysis clearly explains why the effect of neutron rearrangement is negligible in the above mentioned systems (Fig. 2, 3, 4), while it is well-pronounced for the "similar" ${ }^{40} \mathrm{Ca}+{ }^{96} \mathrm{Zr}$ combination. The correlation between the properties of their collective excitations (Tab. 1) and the observed fusion enhancement is clearly seen. The smaller is the rms deformation parameter (and the higher is the energy of the first vibrational state) the smaller effect is expected from the coupling with collective states, and, hence, the larger is an influence of neutron rearrangement on sub-barrier fusion. Additional remark is required concerning octupole vibrational states in the studied systems. For example, ${ }^{96} \mathrm{Zr}$ and ${ }^{100} \mathrm{Mo}$ have strong coupling with octupole vibrations with rather similar properties. These additional excitation modes are effectively taken into account here by fitting the parameters of the ECC model (without neutron rearrangement) to the QCC calculations. However for the particular case namely the difference in the quadrupole properties determines the difference in the magnitude of the fusion enhancement due to neutron rearrangement.

\subsection{Neutron binding energies}

In order to clarify the role of the neutron binding energies we performed the following calculations. The binding energies of two valence neutrons were varied simultaneously in the target and projectile preserving all the other properties ( $Q$ values, potentials, etc.) unchanged. In what follows we will discuss the neutron binding energies in the "donor" nucleus only. The influence of the neutron binding energies on the sub-barrier fusion of light weakly bound nuclei is shown in Fig. 6. One may see that at energies $\sim 3 \mathrm{MeV}$ below the barrier the total effect of neutron rearrangement constitutes about two orders of magnitude (the solid curve as compared to the dotted one). If in the calculations one assumes neutrons in ${ }^{6} \mathrm{He}$ to be more bound (as in ${ }^{9} \mathrm{Li}$ ) but all other properties keeps unchanged, then the effect reduces to one order of magnitude. However in sub-barrier fusion of heavy nuclei the binding energy of transferred neutron has almost no impact on the fusion enhancement! One may see in Fig. 7 that in the case of ${ }^{40} \mathrm{Ca}+{ }^{96} \mathrm{Zr}$ the sub-barrier fusion cross sections shift only a little if one assumes neutrons in ${ }^{96} \mathrm{Zr}$ less bound (as in ${ }^{6} \mathrm{He}$ ) or twice stronger bound (as in ${ }^{16} \mathrm{O}$ ).

The reason why the neutron binding energy plays more important role in fusion of light nuclei becomes clear from Fig. 8, where two nucleus-nucleus potentials are shown for the ${ }^{6} \mathrm{He}+{ }^{64} \mathrm{Zn}$ and ${ }^{40} \mathrm{Ca}+{ }^{96} \mathrm{Zr}$ systems. For the lighter projectile the Coulomb barrier is lower but wider and the classical turning point corresponds to larger distance between nuclear surfaces than for the heavier one due to the smaller $Z_{1} Z_{2}$ Coulomb factor. This discrepancy increases with decreasing energy below the Coulomb barrier. The neutron binding energy determines the "compactness" of its wave function and hence the neutron transfer probability, which is also dependent on the position of the turning point. Thus, the neutron transfer probability decreases much faster with increasing the binding energy in the case 


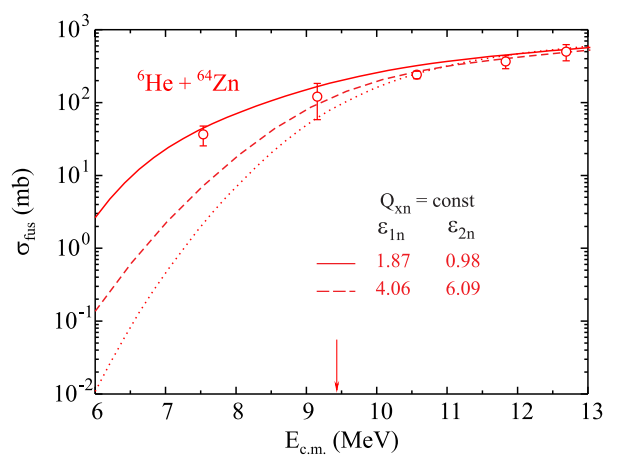

Figure 6. Fusion cross sections for ${ }^{6} \mathrm{He}+{ }^{64} \mathrm{Zn}$ reaction. The data (symbols) are taken from Ref. [10]. The curves are the ECC calculations with (solid and dashed) and without (dotted) neutron rearrangement. The solid curve corresponds to real neutron binding energies in ${ }^{6} \mathrm{He}$. The dashed curve shows the model calculations assuming larger neutron binding energies in ${ }^{6} \mathrm{He}$. The binding energies are shown in $\mathrm{MeV}$.

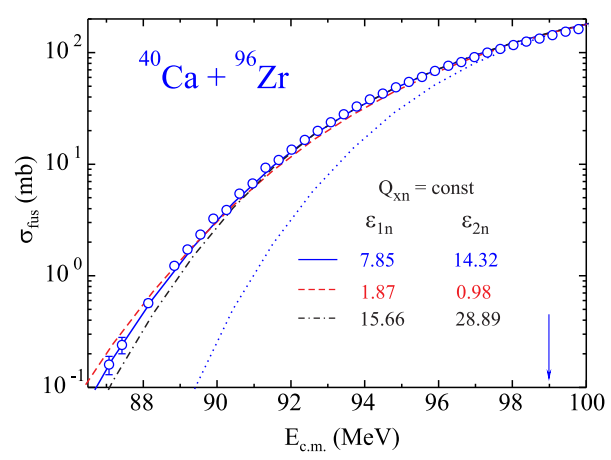

Figure 7. Fusion cross sections for ${ }^{40} \mathrm{Ca}+{ }^{96} \mathrm{Zr}$ reaction. The data (symbols) is taken from Ref. [8]. The curves are the ECC calculations with (solid, dashed, and dash-dotted) and without (dotted) neutron rearrangement. The solid curve corresponds to real neutron binding energies in ${ }^{96} \mathrm{Zr}$. The dashed and dashdotted curves show the model calculations assuming smaller and larger neutron binding energies in ${ }^{96} \mathrm{Zr}$, respectively. The binding energies are shown in $\mathrm{MeV}$.

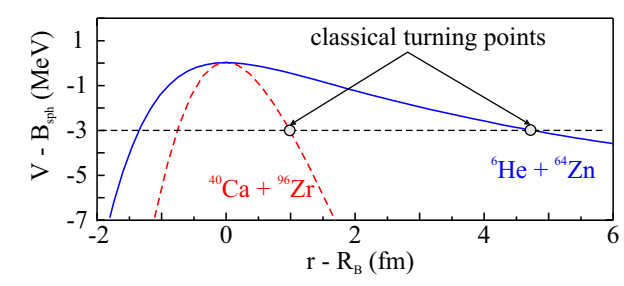

Figure 8. The nucleus-nucleus potential energies for the ${ }^{6} \mathrm{He}+{ }^{64} \mathrm{Zn}$ and ${ }^{40} \mathrm{Ca}+{ }^{96} \mathrm{Zr}$ systems and classical turning points at $E_{\text {c.m. }}=B-3 \mathrm{MeV}$.

of lighter nuclei. This explains why the largest enhance- ment of the sub-barrier fusion cross sections owing to neutron rearrangement is expected (and observed experimentally) for the fusion of light weakly bound nuclei having large positive $Q$ values for neutron transfer.

\section{Conclusions}

The role of neutron rearrangement channels in near-barrier fusion reactions is studied within the ECC model. It is shown that the model reproduces available experimental data. In contrast with the generally accepted opinion, we found that the positive $Q$ values for the neutron transfer is not the only factor determining enhancement of sub-barrier fusion probability. A noticeable additional enhancement of sub-barrier fusion cross section (beside those caused by coupling to collective degrees of freedom) can be expected if the following conditions are fulfilled:

(i) The system has large positive $Q$ values for transfer of one or/and two neutrons. The role of $x n$-channels is negligible for $x>4$.

(ii) The coupling to the collective states is not so much important at sub-barrier energies. This is always the case when one of the reaction partners is a light nucleus. For heavier systems the strongest effect from neutron rearrangement one should expect for spherical nuclei having magic or nearly magic numbers of protons or/and neutrons.

(iii) The valence neutrons are weakly bound (important only for the fusion of light nuclei).

The authors are indebted to the RFBR for support of these studies (Grant Nr. 13-07-00714-a).

\section{References}

[1] K. Hagino, N. Rowley, and A. T. Kruppa, Comput. Phys. Commun. 123, 143 (1999).

[2] V. I. Zagrebaev and V. V. Samarin, Phys. At. Nucl. 67, 1462 (2004).

[3] Fusion code of the NRV, http://nrv.jinr.ru/.

[4] V. I. Zagrebaev, Phys. Rev. C 64, 034606 (2001).

[5] V. I. Zagrebaev, Phys. Rev. C 67, 061601 (2003).

[6] V. I. Zagrebaev, V. V. Samarin, and Walter Greiner, Phys. Rev. C 75, 035809 (2007).

[7] C. Simenel, A. Wakhle, and B. Avez, J. Phys.: Conf. Ser. 420, 012118 (2013).

[8] H. Timmers, et. al, Nucl. Phys. A633, 421 (1998).

[9] A. M. Stefanini, et. al, this conference.

[10] V. Scuderi, et. al, Phys. Rev. C 84, 064604 (2011).

[11] Z. Kohley, et. al, Phys. Rev. Lett. 107, 202701 (2011).

[12] H. M. Jia, et. al, Phys. Rev. C 86, 044621 (2012).

[13] A. M. Stefanini, et. al, Eur. Phys. J. A 49, 1 (2013).

[14] M. Trotta, et. al, Phys. Rev. C 65, 011601 (2001). 